https://idp.uoc.edu

ARTÍCULO

\title{
COVID-19, alquiler turístico y políticas de cancelación ¿emergencia en tiempos de pandemia de la oculta(da) naturaleza de las plataformas digitales?*
}

\author{
Apol·lònia Martínez Nadal \\ Universidad de las Islas Baleares
}

Fecha de presentación: octubre de 2020

Fecha de aceptación: febrero de 2021

Fecha de publicación: marzo de 2021

\section{Resumen}

La naturaleza jurídica de las plataformas digitales es una cuestión polémica y debatida. En el caso de las plataformas de comercialización de estancias turísticas breves, el Tribunal de Justicia de la Unión Europea se ha pronunciado sobre la condición de Airbnb declarando que tiene la sola condición de prestador de servicios de la sociedad de la información a los efectos de la Directiva de comercio electrónico, con las consecuencias que de ello se derivan. Consideramos que este pronunciamiento es cuestionable y revisable pues existen elementos que permiten considerar que esta plataforma tiene un control o influencia decisivos que la alejan de tal neutralidad de mero intermediario tecnológico. En concreto, consideramos que la política de cancelación aplicada por Airbnb en estos tiempos de pandemia, de forma unilateral, es, junto con otros elementos de su modelo negocial, una manifestación de tal posición de control o influencia decisivos, en la medida que decide sobre la existencia y continuidad de una relación de la que pretende ser ajena. En el fondo, se plantea la insuficiencia de los conceptos jurídicos tradicionales y la necesidad de nuevas categorías jurídicas para abordar el tratamiento jurídico de las plataformas digitales.

* Trabajo realizado en el marco del Proyecto RTI2018-097225-B-I00 «Plataformas de intercambio electrónico y nuevos modelos económicos disruptivos; problemática jurídica. En particular, el denominado alquiler turístico vacacional» financiado por el Ministerio de Ciencia e Innovación, la Agencia Española de Investigación y Fondos FEDER de la UE. 


\title{
Palabras clave
}

Airbnb, naturaleza jurídica, prestador de servicios de la sociedad de la información, políticas de cancelación por COVID-19

\section{COVID-19, vacation rentals and cancellation policies: is there an emergency, in these times of pandemic, regarding the hidden nature of digital platforms?}

\begin{abstract}
The legal nature of the digital platforms is a controversial and debated issue. In the case of the platforms for the commercialisation of short-term tourist stays, the European Court of Justice has made a pronouncement on the status of Airbnb, declaring that it is only to be classified as an information society service as defined in the e-Commerce Directive, along with the consequences that arise from this. We consider that this declaration is questionable and revisable as factors exist which allow us to consider that this platform has a decisive control or influence, meaning that this view of its neutrality as a mere technological intermediary is way off the mark. Specifically, we believe that the cancellation policy applied by Airbnb in these times of pandemic, in its one-sided nature, together with other elements of its business model, is evidence of said position of decisive control or influence, as it decides on the existence and continuation of a relationship to which it claims to be unconnected. Ultimately, we outline the insufficiency of the traditional legal concepts and the need for new categories in order to address the legal approach to digital platforms.
\end{abstract}

\section{Keywords}

Airbnb, legal nature, information society service, cancellation policies due to COVID-19

* This work was carried out as part of Project RTI2018-097225-B-I00, "Electronic exchange platforms and new disruptive economic models; legal problems. In particular, the short-term vacation rentals", financed by the Ministry of Science and Innovation, the State Research Agency and the EU European Regional Development Fund. 


\section{La pandemia por la COVID-19 y su impacto en el alquiler vacacional}

Como es sabido, la distópica situación de pandemia provocada por la COVID-19 que nos ha tocado vivir, iniciada formalmente con la declaración por parte de la Organización Mundial de la Salud (OMS) el pasado 11 de marzo de 2020 y con final todavía incierto, ha tenido repercusiones no solo sanitarias y de salud pública sino también, entre otras, económicas y sociales, por todos conocidas, debido a las distintas medidas adoptadas para hacer frente a la misma: confinamientos, cierre de fronteras, clausura de establecimientos...

Estas medidas restrictivas han afectado de forma muy especial al sector del turismo, dadas las limitaciones a la circulación de personas que, consiguientemente, afectan a los viajes, junto con medidas como el cierre obligatorio de establecimientos de alojamiento en los momentos iniciales, las restricciones de aforo, o la exigencia de cuarentena o pruebas de diagnóstico a los viajeros procedentes de territorios de riesgo (entre ellos, desafortunadamente, España), en momentos posteriores.

Esta incidencia sobre el turismo ha afectado, como no podía ser de otra forma, al alquiler turístico provocando una debacle a nivel mundial frente a la que han reaccionado las plataformas más paradigmáticas, como Airbnb, que, desde el inicio de la pandemia ha adoptado medidas diversas: publicación de protocolos sanitarios de desinfección de alojamientos o medidas tendentes al cumplimiento de las medidas legales de contención de la COVID-19 (estableciendo máximos de ocupación, suprimiendo los filtros para búsqueda de alojamientos que permitan fiestas), entre otras.

De entre estas actuaciones, nos centrarnos en la relativa a la aplicación por parte de la plataforma Airbnb de una «Política de Causas de Fuerza Mayor relativa al coronavirus (COVID-19)». Consideramos que esta actuación debe ser analizada, como hacemos en el apartado siguiente, y tenida muy en cuenta a fin de determinar el verdadero papel y naturaleza de esta plataforma, cuestión, como veremos en el último apartado, controvertida, incluso pese a la existencia de recientes pronunciamientos judiciales en la materia en el ámbito comunitario.

\section{Políticas de cancelación de Airbnb con ocasión de la pandemia por la COVID-19}

Como hemos señalado, tras el inicio de la pandemia, Airbnb (cuyo ejemplo inicial fue seguido por otras plataformas) adoptó una medida que consideramos muy relevante desde el punto de vista jurídico, a fin de poner de manifiesto la verdadera naturaleza de esta plataforma: la publicación y aplicación de una «Política de Causas de Fuerza Mayor relativa al coronavirus (COVID-19)» que permite la cancelación de reservas de alojamiento por causas relacionadas con la nueva situación de emergencia sanitaria mundial.

De entrada, llama la atención su carácter unilateral, pues es creada, publicada, aplicada e impuesta por Airbnb, sin previa consulta ni comunicación a los propietarios afectados, siendo, como es, una decisión que tiene una innegable y relevante repercusión (si no se trata directamente de una injerencia) en la relación contractual bilateral entre propietarios arrendadores y clientes arrendatarios («anfitriones» y «usuarios» en la terminología de Airbnb); relación respecto de la que, paradójicamente, la plataforma pretende ser totalmente ajena (como establece en sus Términos de uso)'

Así, de forma general, y antes del inicio de la excepcional situación de crisis sanitaria, según consta en la web de la plataforma: «En Airbnb, los anfitriones pueden elegir qué políticas de cancelación ofrecen a los huéspedes y estos pueden consultarlas antes de efectuar una reserva» ${ }^{2}$. $Y$ se ofrecen de forma predeterminada distintos tipos de cancelación, siendo las ordinarias la flexible, la moderada y la estricta.

1. En efecto, conforme al término 1.2 Airbnb se presenta como mero proveedor tecnológico de la plataforma ajeno a la relación contractual de prestación del servicio de hospedaje entre anfitrión y huésped. En el mismo sentido, el término 7.1.7, relativo al anfitrión.

2. Apartado «Cómo funcionan las cancelaciones de estancias»: https://www.airbnb.es/home/cancellation_policies. 
Pues bien, junto a la política de cancelación ordinaria respecto de la que Airbnb realiza distintas propuestas pero cuya decisión final depende del gestor del alojamiento (que elige dentro de las políticas propuestas por la plataforma), en esta atípica situación de emergencia mundia Airbnb publicó, de forma también atípica y además unilateral, sin siquiera consulta previa a sus «anfitriones» una nueva «Política de Causas de Fuerza Mayor relativa al coronavirus (COVID-19)» que ha pasado a ser de aplicación de forma obligatoria y que rige las relaciones entre arrendador y arrendatario (anfitrión y huésped) respecto de las que, como es sabido, Airbnb se declara completamente ajena en sus «Términos de uso».

Como se explica en la propia web de la plataforma (en la versión actualizada con fecha de 15 de septiembre de 2020), tras la declaración el pasado 11 de marzo de 2020 por la OMS de pandemia mundial por el brote de coronavirus, Airbnb, para hacer frente a la situación, presentó una «Política de Causas de Fuerza Mayor» con cobertura específica para la pandemia con el objetivo de proteger a los miembros de la comunidad. De forma resumida, este es su contenido:

a) Reservas realizadas hasta el 14 de marzo de 2020: están cubiertas las reservas de alojamientos realizadas hasta el 14 de marzo de 2020 y programadas entre el 14 de marzo de 2020 y el 31 de octubre de 2020 y se podrán cancelar antes del comienzo. En consecuencia, los viajeros que cancelen acogiéndose a la política recibirán un reembolso completo. Por su parte, los anfitriones que cancelen en virtud de la política no tendrán que pagar ninguna penalización. Además, los reembolsos y el crédito de viaje que emita Airbnb incluirán todas las comisiones de servicio.

b) Reservas realizadas después del 14 de marzo: en cambio, como señala Airbnb: «Nuestra Política de Causas de Fuerza Mayor no cubrirá las reservas de alojamientos y experiencias en Airbnb realizadas después del 14 de marzo de 2020, excepto en caso de que el viajero o el anfitrión contraigan la COVID-19». La razón alegada es la desaparición de la imprevisibilidad: «Desde que la Organización Mundial de la Salud declaró la COVID-19 como pandemia mundial, no podemos aplicar la Política de Causas de Fuerza Mayor a las cancelaciones relacionadas con el coronavirus y sus consecuencias, puesto que han dejado de considerarse una circunstancia imprevista o inesperada». En esos casos, «aplicaremos la política de cancelación del anfitrión como de costumbre».

c) Reservas de alojamientos realizadas hasta el 14 de marzo de 2020 que tengan una fecha de inicio posterior al 31 de octubre de 2020: no pueden acogerse a la Política de Causas de Fuerza Mayor relativa al coronavirus. En esos casos, «aplicaremos la política de cancelación del anfitrión como de costumbre». No obstante, Airbnb recuerda que está actualizando continuamente la cobertura de esta política por lo que recomienda una consulta periódica de la misma ${ }^{3}$.

Como ya hemos apuntado, esta política de cancelación de reservas (en su versión inicial y sus posteriores actualizaciones) se creó, publicó y aplicó por Airbnb de forma total y absolutamente unilateral, sin acuerdo previo y ni siquiera consulta o comunicación anterior a las principales partes afectadas desde el punto de vista jurídico, anfitrión y huésped (arrendador y arrendatario), y ni siquiera información previa al operador más afectado desde el punto de vista económico (el propietario o gestor de la vivienda), al que ni siquiera se ha exigido aceptación expresa o siquiera tácita de la nueva política de cancelación.

Esta actuación unilateral es reconocida por la propia plataforma: tras publicar la primera versión de la política de cancelación por COVID-19 en marzo de 2020, publicó el día 1 de abril una «Carta a los anfitriones» firmada por Brian Chesky, director ejecutivo de Airbnb, en la que se disculpa formalmente por ello: «Sin embargo, aunque creo que hicimos lo correcto priorizando la salud y la seguridad, quiero disculparme por cómo os comunicamos esta información y por no haber pedido vuestra

3. Esta política se actualiza de forma periódica desde la declaración de pandemia; la última actualización en el momento de redactar este trabajo era la de 15 de septiembre de 2020. Justo al cierre del trabajo se ha publicado una versión con fecha 1 de octubre que amplía la cobertura a las reservas realizadas hasta el 14 de marzo y con fecha de inicio en los próximos cuarenta y cinco días: https://www.airbnb.es/help/article/2701/pol\%C3\%ADtica-de-causas-de-fuerza-mayor-relativa-al-coronavirus\%C2\%A0covid19. 
opinión» ${ }^{4}$. En cualquier caso, esta nueva política ha pasado a ser de aplicación de forma obligatoria y rige las relaciones entre arrendador y arrendatario (anfitrión y huésped), habiendo surgido al margen de los mismos.

Llama la atención que una decisión sobre tal cuestión de tanta trascendencia contractual, que afecta a la existencia y continuidad del contrato bilateral entre anfitrión y usuario (del que, no se olvide, Airbnb no forma parte de acuerdo con su modelo económico y jurídico oficial) sea tomada, decidida e impuesta por una persona ajena a dicha relación. A no ser que realmente no sea tan ajena; en suma, que tal elemento de ajenidad no esté presente o al menos no con tanta pureza e intensidad como se pretende, por ostentar la plataforma una posición de control de tal relación en aspectos tan esenciales como el de su cancelación, posición de control o influencia decisiva, como veremos, especialmente relevante para la determinación de la naturaleza de la plataforma conforme a los postulados de la Unión Europea. En el fondo, sería una muestra de la insuficiencia de esta construcción contractual bilateral para hacer frente a una relación materialmente triangular en la que la plataforma tiene un papel especialmente relevante, tan relevante que se convierte en «regulador» de la misma.

Podría argumentarse que tal actuación la ha realizado la plataforma para hacer frente, de forma ágil y aplicando soluciones uniformes a todos sus «anfitriones» y «huéspedes», a la problemática derivada de la crisis sanitaria. Lo cierto es que, pese a que este objetivo pudiera ser loable desde el punto de vista material, a efectos de agilización de la resolución de estas incidencias en ese atípico momento, se hubiera debido realizar respetando la formal autonomía de la voluntad de las partes contractuales, muy especialmente del anfitrión, que puede ser el más perjudicado económicamente por dicha política de cancelaciones. A no ser, nuevamente, que nos hallemos realmente ante una relación triangular sin el adecuado tratamiento jurídico hoy día.

Como planteamos a continuación, esta decisión de Airbnb sobre políticas de cancelación en tiempos de pandemia debe ser tenida muy en cuenta en el esclarecimiento de la naturaleza de esta plataforma, cuestión, como veremos seguidamente, controvertida, incluso pese a la existencia de recientes pronunciamientos judiciales, como la Sentencia del Tribunal de Justicia de la Unión Europea (TJUE) de 19 de diciembre de 2019 (caso Airbnb Ireland), cuyo planteamiento consideramos revisable.

\section{Naturaleza jurídica de las plataformas digitales: estado de la cuestión. En especial el caso de Airbnb}

Como es sabido, y antes de la crisis generalizada causada por la COVID-19, en los últimos años el alquiler turístico de viviendas había experimentado un gran desarrollo no solo en España y sus comunidades y ciudades más turísticas sino también en otros muchos países y ciudades emblemáticas.

No es un fenómeno nuevo: desde hace años existe este tipo de actividad, tradicionalmente gestionada de forma directa entre particulares o, a lo sumo, a través de agencias tradicionales. El elemento novedoso, y en buena medida causante del mencionado auge, es la aparición de plataformas digitales especializadas que, de forma simple, permiten no solo a profesionales sino también a particulares ofertar una vivienda para su utilización temporal con fines normalmente turísticos en multitud de países y a una gran variedad de potenciales usuarios a los que de otra forma probablemente no podrían Ilegar. Consideramos importante remarcar este papel de las plataformas como factor clave y esencial de esta eclosión del alquiler turístico a nivel mundial y probablemente instrumento imprescindible para determinados arrendadores.

La plataforma paradigmática es, sin duda, Airbnb, surgida en 2008 en San Francisco como iniciativa de unos, por entonces, estudiantes (Brian Chesky, Joe Gebbia y Nathan Blecharczyk) y que se ha convertido hoy en un gigante económico con una amplia oferta de inmuebles en casi doscientos países. Aunque Airbnb sea probablemente la más conocida, existen otras y numerosas plataformas para la comercialización de estancias breves: entre ellas,

4. Véase en este sentido la «Carta a los anfitriones»: https://news.airbnb.com/es/carta-a-los-anfitriones/. 
Booking.com, HomeAway o TripAdvisor. No obstante, el modelo económico no es siempre totalmente coincidente; así, por ejemplo, mientras Airbnb cobra una comisión («tarifa de servicio») sobre el precio del alojamiento reservado, HomeAway aplica un modelo de suscripción del propietario arrendador de tal forma que este debe pagar una tasa anual por la inclusión de su propiedad en la plataforma.

Algunas de estas plataformas digitales pretenden incardinar su modelo de negocio en el ámbito de la denominada «economía colaborativa» o «economía compartida», concepto actualmente amplio, difuso, ambiguo y objeto de tales usos y abusos que ha sido calificado más bien como un «anticoncepto», pues, en efecto, y, paradójicamente, no se ha conseguido todavía una noción compartida de lo que sea dicha «economía compartida» ${ }^{5}$.

En este sentido, consideramos significativo que Airbnb se presente actualmente como simple proveedor de una plataforma tecnológica que actúa como simple mercado en línea, punto de encuentro que posibilita que los «anfitriones» (propietarios, arrendadores) puedan ofrecer sus viviendas a los «huéspedes» (futuros usuarios) ${ }^{6}$. Llama la atención, de entrada, la terminología utilizada (anfitriones, huéspedes), que huye de las categorías jurídicas establecidas (propietario arrendador, cedente; cliente arrendatario, cesionario, usuario) y, sobre todo, de las consecuencias jurídicas que se derivan de las mismas para entrar en ese «limbo jurídico» que se pretende por algunos operadores económicos que sea la economía colaborativa. Y más Ilama la atención todavía que esta terminología sea el resultado de un cambio en los términos de uso de la plataforma producido en el año 2016, en que publica una nueva versión en la que se elimina toda referencia a términos (y conceptos) como alquiler, subarriendo o inmobiliarios, u otras palabras derivadas. Lo cual afecta a la propia definición de la plataforma y la descripción de su actividad, que deja de ser «alquilar» para pasar a ser «anunciar y reservar»» ${ }^{7}$.

$Y$ es que, si las palabras en general no son inocuas, en este caso, visto el trasfondo, lo son menos que nunca. Esta pretensión de huir de las categorías jurídicas tradicionales y de ubicarse confortablemente en el ámbito de la economía colaborativa y de la simple intermediación tecnológica neutral es seguramente interesada y del todo cuestionable por los intereses espurios que se hallan tras la misma y que nos llevan a cuestionar el propio concepto actual de economía colaborativa y la utilización que del mismo se realiza; y ello nos conduce a la búsqueda de términos y categorías más conformes a la realidad económica de estos nuevos modelos negociales disruptivos como, por ejemplo, economía de plataformas o economía digital, entre otros. Todo lo cual obliga a replantear la verdadera naturaleza jurídica de estas grandes plataformas digitales como grandes intermediarias que abren nuevos e inmensos mercados a los propietarios de viviendas a la vez que plantean nuevas cuestiones e incógnitas desde el punto de vista jurídico.

\subsection{Modelo económico y clasificación dual}

Para ello, y para conocer el estado de la cuestión sobre la naturaleza jurídica de las plataformas digitales, podemos tomar como punto de partida que, tradicionalmente, se han distinguido dos modelos económicos con distintas consecuencias jurídicas en materia de obligaciones y responsabilidad, especialmente por la ejecución de la

5. A los efectos de la Comunicación titulada «Una Agenda Europea para la economía colaborativa», presentada por la Comisión Europea en junio de 2016, el término «economía colaborativa» se refiere a modelos de negocio en los que se facilitan actividades mediante plataformas colaborativas que crean un mercado abierto para el uso temporal de mercancías o servicios ofrecidos a menudo por particulares. Por lo general, las transacciones de la economía colaborativa no implican un cambio de propiedad y pueden realizarse con o sin ánimo de lucro.

6. En efecto, conforme al término 1.1 la plataforma es un mercado electrónico, un punto de encuentro virtual entre anfitriones y huéspedes: «La Plataforma de Airbnb es un mercado en línea que permite que los usuarios registrados ["miembros"] y ciertos terceros que ofrecen servicios [los miembros y terceros que ofrecen servicios son "anfitriones" y los servicios que ofrecen son "Servicios de Anfitrión"] publiquen dichos Servicios de Anfitrión en la Plataforma de Airbnb ["Anuncios"] y comuniquen y gestionen directamente con los miembros que desean reservar dichos Servicios de Anfitrión [los miembros que utilizan los Servicios de Anfitrión son "huéspedes"]. Y conforme al término 1.2 es un mero proveedor tecnológico de la plataforma: “En calidad de proveedor de la Plataforma de Airbnb, Airbnb no posee, crea, vende, revende, suministra, controla, gestiona, ofrece, entrega ni presta ningún Anuncio ni Servicio de Anfitrión ni constituye un organizador o minorista de viajes combinados, de conformidad con la Directiva (UE) 2015/2302».

7. Cfr. respecto de este cambio, MORELL RAMOS, J. (2016). «Así se "reinventa" Airbnb en sus nuevos términos y condiciones: https://terminosycondiciones.es/2016/11/14/asi-airbnb-reinventa-nuevos-terminos-y-condiciones/. 
relación subyacente: las plataformas puras, meramente intermediadoras, o las plataformas que, yendo más allá, tienen el papel de prestador de la obligación derivada de la relación subyacente (o, como mínimo, responsable de la misma). Jurisprudencialmente se ha entendido que BlaBlaCar sería un mero intermediario (un prestador de servicios en línea que se dedica a explotar su plataforma y que no tiene la condición de transportista) ${ }^{8}$, mientras que Uber, en cambio, sí tendría la condición de empresario del sector del transporte (es considerado un servicio de intermediación indisociablemente vinculado a un servicio de transporte y, por lo tanto, ha de calificarse de «servicio en el ámbito de los transportes») $)^{9}$.

Para el caso que nos ocupa, en fecha de 19 de diciembre de 2019 se ha pronunciado el Tribunal de Justicia de la Unión Europea (C-390/18) en el sentido de que procede calificar de «servicio de la sociedad de la información» comprendido en el ámbito de aplicación de la Directiva 2000/31 un servicio de intermediación como el de Airbnb, prestado a cambio de una remuneración, que tiene por objeto poner en contacto mediante una plataforma electrónica a potenciales arrendatarios con arrendadores, profesionales o no profesionales, que proponen servicios de alojamiento de corta duración y que, además, ofrece otras prestaciones accesorias de ese servicio de intermediación. Por tanto, entiende el Tribunal que Airbnb es un simple intermediario que no interviene en la prestación del servicio de alojamiento, siendo por ello un mero «prestador de servicios de la sociedad de la información».

En el fondo, la pretensión de acogerse y beneficiarse de la condición de simple intermediario digital, esto es, en términos jurídicos, de «prestador de servicios de la sociedad de la información», es una cuestión estratégica para estas plataformas porque de ella se deriva la aplicación de un marco jurídico y legal que les resulta sumamente favorable.

Así, a modo de ejemplo, la Directiva 2000/31 de comercio electrónico, y los relevantes principios establecidos en la misma tales como la denominada «cláusula del mercado interior» (el prestador está sujeto a la ley del país en el que está establecido, cláusula aplicada a Airbnb Ireland en la mencionada Sentencia del TJUE de 19 de diciembre de 2019); el principio de no autorización previa (no exigencia de autorización específica para los prestadores de servicios de la sociedad de la información, principio objeto de análisis también en esta última sentencia); o la eventual inexistencia de responsabilidad de los intermediarios por los contenidos de la plataforma (cuestión sumamente polémica en los últimos tiempos en derecho español a raíz de la exigencia de responsabilidad a las plataformas de alquiler turístico por la inclusión de viviendas sin el correspondiente número de registro y resuelta de forma divergente por nuestra jurisprudencia).

Pues bien, pese al pronunciamiento del Tribunal de Justicia de la Unión Europea de 19 de diciembre de 2019 en el sentido de que Airbnb es un mero «prestador de servicios de la sociedad de la información», entendemos que, a la vista del funcionamiento y actuaciones de Airbnb, existen argumentos para sostener y considerar que no es realmente tal simple prestador y que, por ello, no podría beneficiarse del régimen jurídico favorable derivado de tal condición.

Veamos a estos efectos con mayor detalle la posición comunitaria en la materia y cómo de la misma pueden extraerse tales argumentos para negar, o como mínimo cuestionar, dicha condición de simple prestador a Airbnb, pese a que el Tribunal europeo haya llegado a la conclusión contraria.

8. En este sentido, existen en España distintos pronunciamientos judiciales: así, por ejemplo, la Sentencia de la Audiencia Provincial de Madrid de 2 de febrero de 2017, que proclama la condición de la plataforma de prestador de servicios de la sociedad de la información a los efectos de la Ley 34/2001; y también la Sentencia de la Audiencia Provincial de Madrid, de 18 de febrero de 2019, en la que se concluye que la actividad de BlaBlaCar no es la de un servicio de transporte, sino la de prestación de servicios en línea, la explotación de una plataforma web.

9. La sentencia del TJUE de 20 de diciembre de 2017 (asunto C-434/15) declaró que «un servicio de intermediación (...) que tiene por objeto conectar mediante una aplicación para teléfonos inteligentes, a cambio de una remuneración, a conductores no profesionales que utilizan su propio vehículo con personas que desean efectuar un desplazamiento urbano, está indisociablemente vinculado a un servicio de transporte, y por lo tanto ha de calificarse de servicio en el ámbito de los transportes (...). En consecuencia, un servicio de esta índole está excluido del ámbito de aplicación del artículo 56 TFUE, de la Directiva 2006/123 y de la Directiva 2000/31». Este mismo criterio resolutivo ha sido aplicado en España por el Tribunal Supremo, en Sentencias de 24 y 25 de enero de 2018, señalando que Uber: «No es un mero servicio de intermediación, sino que constituye una parte sustancial de la prestación de servicio de transporte de viajeros, estando por ello sujeta a la autorización exigida en el artículo 42.1 de la Ley 16/1987, de 30 de julio, de Ordenación de los Transportes Terrestres». 


\subsection{La posición comunitaria. El criterio del control o influencia decisiva y su aplicación a la «Política de Causas de Fuerza Mayor relativa al coronavirus (COVID-19)» de Airbnb, entre otros elementos}

La Comisión Europea presentó en junio de 2016 una Comunicación titulada «Una Agenda Europea para la economía colaborativa» en la que se establecen una serie de pautas que han sido seguidas después, en buena medida, por el Tribunal de Justicia de la Unión Europea:

1. En la Comunicación se establece que en la medida en que las plataformas colaborativas proporcionan un «servicio prestado normalmente a cambio de una remuneración, a distancia, por vía electrónica y a petición individual de un prestatario de servicios $\rangle^{10}$, ofrecen un servicio de la sociedad de la información, por lo que no pueden estar sujetas a autorizaciones previas o cualquier requisito equivalente dirigidos específica y exclusivamente a dichos servicios" ${ }^{11}$.

2. Sin embargo, la misma Comisión admite que puede haber casos en los que plataformas colaborativas ofrecen otros servicios además de los servicios de la sociedad de la información. En particular, en determinadas circunstancias, una plataforma puede ser también un proveedor del servicio subyacente (por ejemplo, servicio de transporte o de alquiler a corto plazo). En tal caso, las plataformas colaborativas podrían estar sujetas a la normativa sectorial específica, incluidos los requisitos de autorización y concesión de licencias empresariales aplicados por lo general a los prestadores de servicios. Obsérvese que la Comisión se mantiene en la tradicional clasificación dual de la naturaleza de las plataformas: o son intermediarias puras o son prestadoras del servicio subyacente, clasificación que actualmente consideramos insuficiente y superada por la realidad económica actual y los nuevos modelos económicos disruptivos. Propugnamos, por ello, la superación de la misma y la aparición de una nueva categoría jurídica que resulte aplicable a las plataformas digitales.
3. En estas coordenadas de la tradicional clasificación dual, la Comisión señala que, normalmente, deberá establecerse caso por caso si una plataforma colaborativa ofrece también el servicio subyacente. $Y$ apunta a que varios elementos desempeñan un papel a este respecto. Así, el nivel de control o influencia que la plataforma colaborativa ejerce sobre el prestador de dichos servicios tendrá por lo general una importancia significativa. $Y_{1}$ en particular, este nivel de control o influencia, según la Comisión, puede establecerse a la luz de los siguientes criterios clave:

a) Precio: ¿fija la plataforma colaborativa el precio final que debe pagar el usuario como beneficiario del servicio subyacente?

b) Otras condiciones contractuales clave: ¿establece la plataforma colaborativa términos y condiciones distintos del precio que determinan la relación contractual entre el prestador de los servicios subyacentes y el usuario?

En este apartado, incluye la Comisión, a título de ejemplo, la existencia de instrucciones obligatorias sobre la prestación del servicio subyacente, incluida cualquier obligación de prestar el servicio. Por nuestra parte, apuntamos esta cuestión para el caso que nos ocupa: ¿acaso el establecimiento unilateral de la política de cancelación del contrato de alojamiento entre huésped y anfitrión por parte de Airbnb no es la fijación de una cuestión contractual clave, un término o condición distintos del precio «que determinan la relación entre el prestador de los servicios subyacentes y el usuario», tanto que de ella depende la existencia y continuidad misma del contrato bilateral, del que ni siquiera es parte la plataforma, conforme a sus «Términos de uso»?

c) Propiedad de activos clave: ¿posee la plataforma activos clave para prestar el servicio subyacente? Como sabemos, Airbnb no es titular de los activos (viviendas) que permiten prestar el servicio de alojamiento; lo que caracteriza a su modelo económico

10. Véase el art. 2, letra a) de la Directiva sobre el comercio electrónico; y el art. 1, apdo. 1, letra b), de la Directiva 2015/1535. También el anexo I de esta última Directiva para una lista indicativa de los servicios no cubiertos por esa definición.

11. Véase el art. 4 de la Directiva sobre el comercio electrónico. 
es, precisamente, la inexistencia de ese tipo de activos empresariales; sin embargo, la plataforma en sí es un activo que resulta, para muchos usuarios, imprescindible para la oferta y contratación del servicio de alojamiento vacacional, pues, de otra forma no podría llegar su oferta a tantos potenciales clientes.

Pues bien, cuando se cumplen estos criterios, según la posición de la Comisión, hay indicios claros de que la plataforma ejerce una influencia o control significativos sobre el prestador del servicio subyacente, lo que puede indicar a su vez que debe considerarse que presta también el servicio subyacente (además de un servicio de la sociedad de la información). Como ya hemos señalado, la Comisión se mueve en los parámetros de la clasificación dual tradicional (prestadores meramente intermediarios y prestadores del servicio subyacente) pero la realidad y los modelos económicos son tan complejos y variados que, como venimos señalando, posiblemente sea conveniente la existencia de una tercera (o simplemente una nueva) categoría con un régimen jurídico propio y diferenciado, en materia de acceso, responsabilidad por la prestación subyacente, responsabilidad por contenidos, implicaciones fiscales, laborales... De tal forma que, en el ámbito de esta tercera o nueva categoría, un prestador puede no ser necesariamente un prestador del servicio subyacente, pero tampoco un simple intermediario, formando parte de una categoría intermedia con un régimen jurídico diferenciado en un mayor o menor número de aspectos y más ajustado y conforme al modelo económico real, en función de la mayor o menor influencia de la plataforma.

En cualquier caso, obsérvese que el criterio de la fijación de una condición contractual esencial como la política de cancelación analizada es muestra de posición de control de la plataforma, como lo son también, entendemos, otros elementos derivados del modelo económico de la misma y que se derivan de los términos de uso que rigen su relación con «anfitriones»y «huéspedes», elementos que ponen de manifiesto que Airbnb no es un simple intermediario tecnológico neutral ajeno a la relación contractual bilateral de arrendadores y arrendatarios.

En efecto, más allá de la alegada condición de mero proveedor tecnológico de una plataforma que es punto de encuentro entre propietarios y usuarios resulta que 1)
Airbnb impone la forma y condiciones de pago del precio del hospedaje e incluso retiene la cantidad pagada en su poder hasta el inicio del servicio de alojamiento; 2 ) influye en la forma y la presentación de los anuncios; 3) puede cancelar reservas; 4) e incluso la configuración de su modelo de «tasas de servicio» se asemeja más al modelo de comisionista (comisionista impuro, por su posición dominante) que recibe en pago una comisión por reserva realizada que al modelo de simple alojador de datos («tablón de anuncios») que cobra por el simple anuncio, con independencia de las reservas posteriores; 5) por ello, más allá de un simple alojador de contenidos, la propia plataforma está interesada en promocionar las reservas, ya que su remuneración depende de la efectiva reserva, no de la simple inclusión del anuncio.

En suma, tras este análisis del trasfondo económico de la relación materialmente triangular entre Airbnb y sus usuarios (anfitrión y huésped), y conforme a los criterios de la propia Comisión, consideramos que existen elementos acreditativos suficientes de la posición de control o influencia de la plataforma que permiten cuanto menos cuestionar su condición de mero prestador de servicios de la sociedad de la información con funciones de intermediación neutral.

Estos criterios derivados de la Comunicación de la Comisión Europea «Una Agenda Europea para la economía colaborativa» establecidos en 2016 que acabamos de exponer de forma resumida han sido seguidos después, en buena medida, por el Tribunal de Justicia de la Unión Europea. En concreto, y por lo que nos interesa a efectos de este trabajo, hemos de volver a la ya conocida Sentencia del TJUE de 19 de diciembre de 2019 (Asunto C-390/118) que, como es sabido, se pronuncia por primera vez sobre la naturaleza jurídica de la plataforma Airbnb.

Recordemos que la sentencia declara que el servicio prestado por Airbnb Ireland debe entenderse como un «servicio de la sociedad de la información» en el sentido de la Directiva 2000/31 de Comercio Electrónico. A continuación, el Tribunal declara que por ello no es conforme a la Directiva que Francia exija a Airbnb Ireland contar con una autorización para realizar su actividad, ya que esta prohíbe que los Estados impongan restricciones a los operadores establecidos en otros Estados miembros (Airbnb Ireland está establecido en Irlanda), a menos que hayan 
tramitado previamente un procedimiento previsto en el artículo 3.4 de la propia Directiva, el cual requiere que se haya notificado al Estado de establecimiento del operador y a la Comisión.

La sentencia dedica apenas el apartado 68 al examen de la existencia de una posición de control y de influencia decisiva, un examen breve y sobre una base fáctica incompleta que no refleja el verdadero modelo negocial de Airbnb y que le lleva a descartar tal posición. Mayor extensión hallamos en la Posición del Abogado General, con los apartados 69 a 79 dedicados al análisis del control o influencia decisiva ejercidos sobre las condiciones de la prestación de servicios; especialmente interesante a nuestros efectos es el párrafo 74 en el que se señala: «es preciso observar que son los arrendadores quienes fijan las condiciones del alquiler. Es cierto que AIRBNB Ireland establece determinadas opciones predefinidas en cuanto a las condiciones de cancelación. Sin embargo, es, en todo caso, el arrendador quien elige, de manera deliberada, una de las opciones propuestas $y_{1}$ por tanto, en quien recae la decisión final sobre las condiciones de cancelación». Como hemos visto, la política de cancelación por causas de fuerza mayor que aplica Airbnb a causa de la COVID-19 impide afirmar que «en todo caso» es del arrendador la decisión final sobre las cancelaciones y muestra la influencia decisiva de la plataforma (incluso antes de la pandemia Airbnb tenía una política de cancelación por fuerza mayor con efectos similares)

Esta sentencia, obviamente, ha sido acogida muy favorablemente por el sector de las plataformas de alojamiento, no solo por el pronunciamiento en sí sino también por las consecuencias jurídicas y prácticas, que pretenden derivarse del mismo, no siempre de forma fundamentada (caso de la exención de responsabilidad por publicación de contenidos).

Pues bien, aunque es cierto que este único pronunciamiento judicial proclama que Airbnb tiene tal condición, consideramos que, a la vista de lo expuesto en este trabajo sobre el modelo económico y la posición de control y de influencia decisiva de esta plataforma sobre el prestador del servicio de hospedaje y su relación contractual con el usuario, no se ha realizado un adecuado análisis de la verdadera naturaleza de la actuación de esta plataforma, de la que las políticas de cancelación por la COVID-19 no son más que «una punta del iceberg» que muestra, siquiera parcialmente, una parte oculta(da) de esta plataforma, más allá de la de un simple intermediario neutral.

\section{Conclusiones}

Resoluciones como la Sentencia del Tribunal de Justicia de la Unión Europea de 19 de diciembre de 2019 calificando a la plataforma Airbnb como mero prestador de servicios de la sociedad de la información conforme a la Directiva de comercio electrónico ponen de manifiesto la dificultad de establecer la naturaleza jurídica de las plataformas digitales.

Beneficiarse de esta calificación es un tema estratégico crucial para las plataformas digitales, pero entendemos que, en la actualidad, esta categoría jurídica no resuelve satisfactoriamente la problemática subyacente a estos nuevos modelos económicos disruptivos.

De lege data, consideramos que es cuanto menos cuestionable, en el supuesto de Airbnb, su calificación como mero prestador de servicios de la sociedad de la información. Actuaciones como la publicación durante la pandemia de la política de cancelación por COVID-19, junto con otros elementos característicos de su actuación, permiten sostener que esta plataforma tiene un control o influencia decisiva sobre las partes de la relación de hospedaje que impiden calificarla como un simple intermediario neutral, mero prestador de servicios de la sociedad de la información, con las consecuencias que de esa condición se derivan.

De lege ferenda, y para abordar de forma adecuada el tratamiento jurídico de las plataformas digitales, consideramos recomendable superar la tradicional clasificación dual de las plataformas (intermediarias vs. ejecutoras) y las actuales categorías establecidas (prestador de servicios de la sociedad de la información) que tuvieron su razón de ser en los momentos iniciales de surgimiento del comercio electrónico pero que resultan inadecuadas para la economía de plataformas. Hay que avanzar hacia nuevas categorías que den una mejor cobertura a estos nuevos modelos económicos disruptivos, como se ha planteado doctrinalmente. Es necesario, pues, establecer nuevos regímenes jurídicos adaptados y adecuados al verdadero papel y funciones de las plataformas digitales. 
En este sentido, puede tomarse como referencia el documento «Model rules on online platforms» presentado por el European Law Institute, en cuyo artículo 18 se regula precisamente la «Responsabilidad del operador de la plataforma con influencia predominante» y se establece como principio general que si el cliente puede confiar razonablemente en que el operador de la plataforma tendrá una influencia predominante sobre el proveedor, «el cliente puede ejercer los derechos y recursos disponibles por el incumplimiento contra el proveedor en virtud del contrato proveedor-cliente también contra el operador de la plataforma». Aparece, pues, también la idea de «influencia predominante», para cuya evaluación se propone considerar distintos criterios, buena parte de los cuales son cumplidos por Airbnb: por ejemplo, conclusión del contrato proveedor-cliente exclusivamente a través de la plataforma; ocultación de la identidad del proveedor o los datos de contacto hasta después de la conclusión del contrato proveedor-cliente; retención por la plataforma de los pagos realizados por el cliente al proveedor; determinación de términos esenciales del contrato por la plataforma; marketing centrado en la plataforma y no en los proveedores. A mayor cumplimiento de estos criterios, existirá mayor influencia decisiva y mayor probabilidad de ejercicio de una acción de responsabilidad contra el intermediario, pese a no ser parte de la relación bilateral cliente-proveedor, bilateralidad que debería ser también superada con el reconocimiento jurídico de la estructura triangular derivada de la economía de plataformas y la posición de predominio de estas.

En suma, estamos en un momento clave en el que se pone de manifiesto la necesidad de superar, replantear y definir nuevas categorías jurídicas para estos nuevos modelos económicos disruptivos.

\section{Adenda}

Una vez finalizada en el mes de octubre de 2020 la redacción de este trabajo, la Comisión Europea presentó el pasado mes de diciembre de 2020 dos propuestas para regular los servicios digitales a través de dos instrumentos: la Propuesta de Reglamento de Mercados Digitales (conocida abreviadamente como DMA por sus siglas en inglés, «Digital Markets Act») y la Propuesta de Reglamento de Servicios Digitales (conocida como DSA, por «Digital Services Act»); ambas forman parte del denominado «Digital Services Act Package» y proponen una nueva regulación aplicable a las plataformas digitales, especialmente las grandes plataformas tecnológicas.

Por ello, en la posterior fase de revisión final del trabajo previa a su publicación, consideramos necesaria como mínimo una breve referencia a estas iniciativas y al tema planteado de la naturaleza jurídica y responsabilidad de las grandes plataformas. Del análisis de estas propuestas, en su estado actual, no se deduce una solución clara para el problema planteado en nuestro trabajo, aun cuando aparecen algunas ideas interesantes; por ejemplo, la posible responsabilidad ex artículo 5, párrafo 3 de la propuesta de Reglamento de Servicios Digitales de las plataformas ante consumidores en el supuesto de que la plataforma permita que la transacción se desarrolle de tal forma que lleve a un consumidor medio y razonablemente bien informado a creer que la información, o el producto o servicio objeto de la transacción, son proporcionados por la propia plataforma en línea o por un destinatario del servicio que actúa bajo su autoridad o control. Es solo un primer paso, en la línea de nuestras propuestas, que consideramos necesitaría una mayor concreción, la cual esperamos se consiga en el largo recorrido que les espera todavía a estas dos relevantes propuestas de Reglamento. 


\section{Referencias bibliográficas}

BUSCH, C.; SCHULTE-NÖLKE, H; WIEWIOROWSKADOMAGALSKA A.; ZOLL, F. (2016). «The rise of the platform economy: A new challenge for EU Consumer Law? EUCML. Journal of European and Consumer Law, pág. 3 y sigs.

CUENA CASAS, M. (2020). «La contratación a través de plataformas intermediarias en línea». Cuadernos de Derecho Transnacional, vol. 12, núm. 2, págs. 283-348 [en línea] DOI: https://doi.org/10.20318/ cdt.2020.5612 [Fecha de consulta: 8 de febrero de 2021].

DE MIGUEL ASENSIO, P. (2019). «La ordenación de las plataformas de intermediación tras la(s) sentencia(s) Airbnb» [en línea] https://pedrodemiguelasensio.blogspot.com [Fecha de consulta: 8 de febrero de 2021].

EUROPEAN COMMISSION (2016). Communication from the Commission to the European Parliament, the Council, the European Economic and Social Committee and the Committee of the Regions, Online Platforms and the Digital Single Market Opportunities and Challenges for Europe, SWD (2016) 172 final, COM (2016) 288 final Brussels, 25 May 2016.

EUROPEAN COMMISSION (2016). Communication from the Commission to the European Parliament, the Council, the European Economic and Social Committee and the Committee of the Regions, European Agenda for the collaborative economy, SWD (2016) 184 final, COM (2016) 356 final Brussels, 2 June 2016.

EUROPEAN LAW INSTITUT (2019). Model rules on online platforms [en línea] https://www.europeanlawinstitute.eu [Fecha de consulta: 8 de febrero de 2021].

FERNÁNDEZ GARCÍA DE LA YEDRA, A. (2020). «Calificación jurídica de las plataformas de alojamiento colaborativo». En: MUNAR, P. (dir.). Turismo, vivienda y economía colaborativa. Pamplona: Aranzadi, págs. 421-446.

JIMÉNEZ HORWITZ, M. (2019). «La situación jurídica de la plataforma Airbnb en el marco de la economía colaborativa». Revista Aranzadi Doctrinal, núm. 3.

MARTÍNEZ NADAL, A. (2020). Alquiler turístico de viviendas de uso residencial y Derecho de la Competencia. Pamplona: Aranzadi.

PAZOS CASTRO, Ricardo (2020). «Uber, Airbnb y la llamada "influencia decisiva" de las plataformas digitales». IDP. Revista de Internet, Derecho y Política, núm. 31, págs. 1-14. UOC [en línea] DOI: https://doi.org/10.7238/idp.v0i31.3224 [Fecha de consulta: 8 de febrero de 2021].

RODRÍGUEZ DE LAS HERAS BALLELL, T. (2017). «The legal anatomy of electronic platforms: A prior study to assess the need of a Law of Platforms in the EU». The Italian Law Journal, vol. 3, núm. 1, pág. 149 y sigs [en línea] https://www.academia.edu/34575454/T_Rodriguez_de_las_Heras_BaIlell_The_Legal_Anatomy_of_Electronic_Platforms_A_Prior_Study_to_Assess_the_Need_of_a_ Law_of_Platforms_in_the_EU [Fecha de consulta: 8 de febrero de 2021].

RODRÍGUEZ RUIZ DE VILLA, D. (2020). «Enseñanzas de la Sentencia Airbnb del TJUE para el corretaje inmobiliario en España» [en línea] https://almacendederecho.org [Fecha de consulta: 8 de febrero de 2021]. 


\title{
Cita recomendada
}

MARTÍNEZ NADAL, Apol·lònia (2021). «COVID-19, alquiler turístico y políticas de cancelación ¿emergencia en tiempos de pandemia de la oculta(da) naturaleza de las plataformas digitales?». IDP. Revista de Internet, Derecho y Política, núm. 32 (marzo). UOC [Fecha de consulta: dd/mm/aa] http://dx.doi.org/10.7238/idp.v0i32.374912

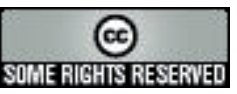

\begin{abstract}
Los textos publicados en esta revista están -si no se indica lo contrario- bajo una licencia Reconocimiento-Sin obras derivadas 3.0 España de Creative Commons. Puede copiarlos, distribuirlos y comunicarlos públicamente siempre que cite su autor y la revista y la institución que los publica (IDP. Revista de Internet, Derecho y Política; UOC); no haga con ellos obras derivadas. La licencia completa se puede consultar en: http://creativecommons.org/ licenses/by-nd/3.0/es/deed.es.
\end{abstract}

\section{Sobre la autora}

Apol·lònia Martínez Nadal

Catedrática de Derecho Mercantil

Universidad de las Islas Baleares

apollonia.martinez@uib.es

Apol·lònia Martínez Nadal, catedrática de Derecho Mercantil de la Universidad de Ias Islas Baleares. Investigadora responsable del CEDIB (Centro de Estudios del Derecho de la Informática de Baleares). Ha sido vocal adscrita de la Comisión General de Codificación para la elaboración de la Propuesta de Código Mercantil de 2013. Entre sus líneas de investigación destacan las relativas al derecho de las nuevas tecnologías y al derecho del turismo. En estos ámbitos es autora de distintas monografías sobre firma electrónica, comercio electrónico, pagos electrónicos o contratos turísticos, entre otras. Ha dirigido y participado en numerosos proyectos de investigación competitivos. Actualmente es investigadora principal del Proyecto de Investigación RTI2018-097225-B-I00 «Plataformas de intercambio electrónico y nuevos modelos económicos disruptivos; problemática jurídica. En particular, el denominado alquiler turístico vacacional». 